\title{
Discriminating malaria and dengue fever in endemic areas: Clinical, biochemical and radiological criteria
}

\author{
Vineet Jain $^{\mathrm{a}}$, Afreen Khan ${ }^{\mathrm{a}, * *}$, Richa Garg ${ }^{\mathrm{b}}$, Arushi Chopra ${ }^{\mathrm{b}}$, Diksha Gaur ${ }^{\mathrm{b}}$, \\ Varun Kumar Kashyap ${ }^{c}$, Sunil Kohli ${ }^{\mathrm{a}}$, Kanupriya Bajaj ${ }^{\mathrm{b}}$ \\ ${ }^{a}$ Department of Medicine, Hamdard Institute of Medical Sciences \& Research (HIMSR), New Delhi, India \\ ${ }^{\mathrm{b}}$ Hamdard Institute of Medical Sciences \& Research (HIMSR), New Delhi, India \\ ${ }^{\mathrm{c}}$ Biostatics, Department of Community Medicine, Hamdard Institute of Medical Sciences \& Research (HIMSR), New Delhi, India
}

\section{A R T I C L E I N F O}

\section{Keywords:}

Malaria

Dengue

Acute febrile illness

Short pyrexia

\begin{abstract}
A B S T R A C T
Background: India bears a high burden of both dengue and malaria. An early distinction between the two could be invaluable in reducing mortality and morbidity. The objective of this study is to identify clinical, biochemical, and radiological parameters for the same, which could aid early detection and treatment of both diseases. Methods: A cross-sectional study was conducted at a tertiary care hospital in South Delhi, India. 100 pre-diagnosed patients each of dengue and malaria were studied. Features on clinical examination, and results of radiological and biochemical investigations were recorded. Continuous variables were compared using $t$-test, whereas categorical variables were subjected to chi-square test. P-value of $\leq 0.05$ was taken as significant.

Results: All patients presented with short pyrexia (fever $<7$ days). Rash (27\%), pruritus (28\%), abdominal pain $(30 \%)$ and breathlessness (20\%) were significantly associated with dengue, whereas headache $(99 \%)$ was significantly associated with malaria. Radiological investigations showed patients with dengue had pleural effusion (46\%) and ascites (49\%), and hepatosplenomegaly was more common in malaria (44\%). Mean hemoglobin levels, and platelet counts were significantly lower in malaria $\left(11.24 \mathrm{~g} / \mathrm{dL}\right.$, and $62.07 \times 10^{3} / \mathrm{mm}^{3}$ respectively), than in dengue (13.03 $\mathrm{g} / \mathrm{dL}$, and $84.61 \times 10^{3} / \mathrm{mm}^{3}$ respectively). Liver enzymes showed rise in both diseases with a marked elevation in dengue. Raised serum bilirubin levels and mild renal dysfunction were both highly significant for malaria $(\mathrm{P}<0.001)$.

Conclusion: The study concludes that a distinction between dengue and malaria can be made on the basis of their clinical, radiological and biochemical findings.
\end{abstract}

\section{Introduction}

With the dawn of the present decade, the focus on prevention of infectious diseases intensified manifold. This is perhaps illustrated best by two World Health Organisation (WHO) initiatives, namely: Global Strategy for Dengue Prevention and Control 2012-2020 ${ }^{1}$ and Global Technical Strategy for Malaria 2016-2030. ${ }^{2}$

Malaria and dengue fever are mosquito borne diseases that lead to short term pyrexia. Dengue fever is a severe, flu-like viral illness. It is caused by the bite of infected female mosquitoes mainly of the species Aedes aegypti and-to a lesser extent- Aedes albopictus, which transmits dengue virus (DENV), a flavivirus. ${ }^{3}$ Malaria is an acute febrile illness caused by Plasmodium parasites that are transmitted to people through the bites of infected female Anopheles mosquitoes. There are five plasmodium species that cause malaria in humans, of which the largest threat is posed by $P$. falciparum and P.vivax. ${ }^{4}$

Globally, 219 million cases of malaria occurred in 2017, with the South-East Asia Region bearing the second highest burden. ${ }^{5}$ On the other hand, a 2013 study estimated there are 390 million infections of dengue annually. ${ }^{6}$ India contributes significantly to these numbers. In 2018, 101,192 cases of dengue had occurred in the country, causing 172 deaths; at the same time, 429,928 cases of malaria had been reported, causing 96 deaths. In 2019, by end of May 5504 cases of dengue, and 66,313 cases of malaria had been recorded by the National Vector Borne Disease Control Programme (NVBDCP). ${ }^{7}$

The clinical manifestations of dengue and malaria are similar i.e.,

\footnotetext{
* Corresponding author.

E-mail addresses: dr.vineet83@yahoo.in (V. Jain), kh.afreen@gmail.com (A. Khan), richagarg.rg96@gmail.com (R. Garg), arushi.chopra96@gmail.com (A. Chopra), diksha.gaur@gmail.com (D. Gaur), varun02stat@gmail.com (V.K. Kashyap), sunil.kohli@gmail.com (S. Kohli), kanupriya193@gmail.com (K. Bajaj).
} 
fever accompanied by non-specific symptoms, which may include headache, arthralgia, myalgia, and gastrointestinal symptoms, ${ }^{8}$ and both are the most common etiologies of short pyrexia in India. ${ }^{9}$ Additionally, both diseases follow seasonal trends, peaking approximately simultaneously during the post monsoon months (June to September). These factors confound the early and precise clinical diagnosis of these vector-borne diseases.

Currently, laboratory diagnosis of dengue is established directly by the detection of viral components[non-structural glycoprotein-1 (NS1) antigen] in serum, or indirectly by the measurement of antibodies $(\operatorname{IgM}){ }^{3}$ Meanwhile, diagnosis of malaria is either by microscopic examination of blood smears, or with Rapid Diagnostic Tests (RDTs). ${ }^{4}$ Microscopic examination raises a need for skilled manpower, which often goes unmet for large number of people living in rural areas, whereas serological tests are often expensive, and consequently, unaffordable. In such resource-poor settings, treatment for malaria may be initiated presumptively which can lead to the emergence of antimalarial drug resistance. ${ }^{10}$ Moreover, both dengue and malaria can lead to life threatening complications, ${ }^{5,6}$ and hence need to be diagnosed early and accurately in order to permit timely management.

Therefore, the objective of this study was to identify clinical and laboratory features that could help us in differentiating between patients infected with dengue and those infected with malaria. It will prevent unnecessary treatments, hospitalisations and interventions. Timely clinical differentiation between dengue and malaria could guide clinicians to identify patients earlier and help prevent complications. Further, accurate diagnosis could facilitate timely public health measures, e.g. vector control targeted at the households of index cases. ${ }^{11}$

Prior to this study, although numerous authors have compared dengue and malaria, ${ }^{8,11,12}$ we could not find any research that used as comprehensive a set of parameters as present in this study.

\section{Methods}

The present cross-sectional study was conducted in the department of Medicine at a tertiary care hospital located in South Delhi, India. Patients were recruited in post monsoon months (July to September) for two consecutive years, 2017 and 2018.

A total of 200 adult patients were included in the study of which 100 patients were pre-diagnosed cases of dengue fever and 100 prediagnosed cases of $P$. vivax malaria.

The sample size was calculated on the basis of a literature report ${ }^{13}$ and using statistical formula. Dengue was diagnosed on the basis of NS1 antigen or IgM antibody positivity determined by ELISA (enzymelinked immunosorbent assay) (Biorad ELISA reader). Similarly, P. vivax malaria was diagnosed using peripheral smear (using Giemsa stain) or malaria antigen rapid diagnostic test. Patients infected with more than one pathogen or with obvious signs of localized infections were excluded from the study.

Informed consent was taken from all patients. Ethical clearance was taken from the Institutional Ethics Committee.

A detailed history was taken; a thorough physical examination was done. All patients were subjected to complete hemogram, liver function test (LFT), kidney function test (KFT), chest radiograph (Siemens Multiselect DR) and ultrasonography (Samsung HS70 5D) of abdomen. Patients' platelet count and liver function tests were recorded daily. The lowest value of hemoglobin and platelet count and the highest values of blood urea, serum creatinine, serum bilirubin, serum glutamic oxaloacetic transaminase (SGOT) and serum glutamic pyruvic transaminase (SGPT) of each patient during the course of hospital stay were recorded. The hematological and biochemical parameters were measured using automated systems (Sysmex Hematology analyzer and Siemens Xpand biochemistry autoanalyzer respectively) at the diagnostic laboratory of the hospital. These parameters were standardized by using internal and external quality control samples, and details were documented in the laboratory. Reference ranges of these investigations
Table 1

Demographics of malaria and dengue patients.

\begin{tabular}{llll}
\hline & & Malaria & Dengue \\
\hline Total Patients & & 100 & 100 \\
Mean Age & 30.37 years & 25.71 years \\
Sex & Males & 70 & 61 \\
& Females & 30 & 39 \\
\hline
\end{tabular}

were defined by the reference ranges of the hospital laboratory. Data was collected and tabulated in excel sheets.

Statistical analysis was performed using SPSS software (SPSS Inc., Released 2017 SPSS for Windows, version 25.0, Chicago, IL, USA). Mean and Standard Deviation (SD) was calculated for the continuous variables, and $t$-test or Mann Whitney $U$-test was used to test the significance. Categorical variables were expressed in proportion and Chisquare test or Fisher Exact test was used to compare dichotomous variables. Their $95 \%$ confidence intervals (CI) were calculated. For all tests, a two sided $\mathrm{P} \leq 0.05$ was considered statistically significant.

\section{Results}

For this study, 100 pre-diagnosed patients each of dengue and malaria (Table 1) were compared on the basis of clinical, biochemical and radiological parameters.

As shown in Table 2, it was found that rash, pruritus, abdominal pain and breathlessness were significant findings for dengue. On the other hand, headache appeared to be significantly more common in malaria.

Analysis of the patients' radiological profiles (Table 3 ) revealed that while ascites and pleural effusion were significantly associated with dengue, neither gallbladder wall edema nor hepatosplenomegaly showed any association with either disease.

Both groups of patients were subjected to thorough laboratory investigations, including complete blood count (CBC), LFT and KFT. An analysis of the results revealed that hemoglobin as well as platelet count had significantly lower mean values in malaria than in dengue. Further, both serum creatinine and serum urea showed significantly ( $P$ value $<0.001$ ) higher mean values in malaria, indicating inadequate kidney function. On the other hand, liver function was found to be deranged much more in dengue as compared to malaria. All parameters-alkaline phosphatase, SGOT, SGPT-showed significantly higher mean values in dengue than in malaria ( $\mathrm{P}$ values $<0.05,<0.001,<$ 0.001 respectively). The mean value of bilirubin was raised more in malaria than in dengue, and this difference was highly significant ( $P$ value $<0.001$ ). A comparison of laboratory findings is charted in Table 4.

\section{Discussion}

In the present study, both diseases generally presented in a young patient age group; with the mean age of dengue patients even lesser than that of malaria patients. This was in concordance with the results

Table 2

Clinical Parameters of malaria and dengue patients.

\begin{tabular}{llll}
\hline & Malaria $(\mathrm{n}=100)$ & Dengue $(\mathrm{n}=100)$ & P Value \\
\hline Fever & 100 & 100 & $\mathrm{NA}$ \\
Rash & 2 & 27 & $\mathrm{P}<0.05$ \\
Bleeding & 17 & 27 & $\mathrm{P}>0.05$ \\
Chills & 80 & 76 & $\mathrm{P}>0.05$ \\
Pruritus & 1 & 28 & $\mathrm{P}<0.05$ \\
Headache & 99 & 46 & $\mathrm{P}<0.05$ \\
Pain abdomen & 12 & 30 & $\mathrm{P}<0.05$ \\
Breathlessness & 4 & 20 & $\mathrm{P}<0.05$ \\
\hline
\end{tabular}


Table 3

Radiological Parameters of malaria and dengue patients.

\begin{tabular}{llll}
\hline & Malaria $(\mathrm{n}=100)$ & Dengue $(\mathrm{n}=100)$ & $\mathrm{P}$ Value \\
\hline Hepatosplenomegaly & 44 & 30 & $\mathrm{P}>0.05$ \\
Ascites & 2 & 49 & $\mathrm{P}<0.05$ \\
Gall bladder wall edema & 12 & 14 & $\mathrm{P}>0.05$ \\
Pleural Effusion & 2 & 46 & $\mathrm{P}<0.05$ \\
\hline
\end{tabular}

Table 4

Laboratory Parameters of malaria and dengue patients.

\begin{tabular}{|c|c|c|c|c|c|}
\hline & \multicolumn{2}{|l|}{ Malaria } & \multicolumn{3}{|l|}{ Dengue } \\
\hline & Mean & Std. Deviation & Mean & Std. Deviation & $\mathrm{P}$ value \\
\hline $\begin{array}{l}\text { Hemoglobin (g/ } \\
\text { dL) }\end{array}$ & 11.246 & 2.6039 & 13.032 & 2.6557 & $<0.001$ \\
\hline $\begin{array}{c}\mathrm{TLC}\left(\mathrm{x} 10^{3} /\right. \\
\left.\mathrm{mm}^{3}\right)\end{array}$ & 5.1017 & 2.20377 & 5.4382 & 3.60586 & $>0.05$ \\
\hline $\begin{array}{l}\text { Platelet count } \\
\qquad\left(\mathrm{x} 10^{3} / \mathrm{mm}^{3}\right)\end{array}$ & 62.07 & 35.57415 & 84.6121 & 59.36873 & $<0.05$ \\
\hline $\begin{array}{l}\text { Serum creatinine } \\
\qquad(\mathrm{mg} / \mathrm{dL})\end{array}$ & 1.0468 & 0.43012 & 0.9248 & 1.04142 & $<0.001$ \\
\hline Urea $(\mathrm{mg} / \mathrm{dL})$ & 33.24 & 17.47617 & 21.1663 & 11.84716 & $<0.001$ \\
\hline ALP (IU/L) & 125.25 & 57.959 & 107.96 & 57.057 & $<0.05$ \\
\hline SGOT (IU/L) & 47.03 & 30.657 & 186.8 & 249.8 & $<0.001$ \\
\hline SGPT (IU/L) & 59.9 & 80.085 & 123.23 & 127.223 & $<0.001$ \\
\hline $\begin{array}{l}\text { Serum bilirubin } \\
(\mathrm{mg} / \mathrm{dL})\end{array}$ & 1.8974 & 1.92549 & 0.7602 & 0.51041 & $<0.001$ \\
\hline
\end{tabular}

of Epelboin et al. ${ }^{8}$ Preponderance of both diseases in males mimics the findings of studies done both globally, ${ }^{8}$ and in India. ${ }^{12}$ This may be a product of higher degree of exposure in males. It may also be contributed to the fact that women in India tend to present less frequently to hospitals.

On examining both patient groups clinically, rash and pruritus were significant features for dengue, while they were seldom present in patients of malaria. Prior studies in North Indian hospitals, ${ }^{14,15}$ have reported even higher percentages of both rash (40\% and $46.8 \%)$ and pruritus ( $50 \%$ and $27.6 \%$ ) in dengue. The WHO too has stated rash as a common symptom in dengue patients. ${ }^{3}$ On the other hand, almost all patients of malaria presented with headache. Epelboin et al. ${ }^{8}$ too had reported a higher incidence of headache in malaria (63.3\%) than dengue (59.2\%). Severe abdominal pain and rapid breathing are generally considered markers for severe dengue. ${ }^{3}$ Further, bleeding-while more common in dengue than in malaria-was not significant for either. This contrasted with the findings of Abhilash et al. ${ }^{12}$ which established bleeding as highly significant $(\mathrm{P}<0.001)$ for dengue when compared with other causes of Acute Undifferentiated Febrile Illnesses (AUFI).

Both groups of patients underwent radiological investigations. Significant findings of ascites and pleural effusion in dengue compared favourably with the results of studies by Nawale et al. ${ }^{16}$ and Motla et al., ${ }^{17}$ and can be explained by the plasma leakage known to occur in dengue. $^{3}$ Prior studies ${ }^{18,19}$ have established the association of splenomegaly with malaria which was also noted in the present study. Abhilash et al. ${ }^{12}$ found hepatic involvement more commonly in malaria (47\%) than dengue $(31 \%)$ which corroborates our findings.

Hematological investigations of malaria and dengue patients were monitored closely. Lower mean hemoglobin levels were found in malaria than in dengue. This was supported by the results of studies by Kotepui et al. ${ }^{20}$ and Abhilash et al. ${ }^{12}$ that determined median hemoglobin of malaria patients ( $12.3 \mathrm{~g} / \mathrm{dL}$ and $12.4 \mathrm{~g} / \mathrm{dL}$ respectively) was lower than that of dengue patients $(13.6 \mathrm{~g} / \mathrm{dL}$ and $14.8 \mathrm{~g} / \mathrm{dL}$ respectively). This can be attributed to the invasion of erythrocytes by Plasmodium leading to hemolysis in malaria. ${ }^{21}$ In our study, a more severe thrombocytopenia was observed in patients with malaria. While surprising, this finding resembles the results of studies by Carme et al. ${ }^{22}$ and Shah et al. ${ }^{23}$

Lastly, the biochemical profiles (LFT and KFT) of both patient groups were documented, which yielded fascinating results. Mild renal dysfunction was documented in patients with malaria more commonly. Prior studies by Epelboin et al. ${ }^{8}$ and Nair et al. ${ }^{24}$ have established malaria as the more common etiology of renal dysfunction, when compared with other tropical acute febrile illnesses. The most commonly accepted hypotheses for such a presentation of renal failure in malaria are mechanical obstruction by infected erythrocytes, immunemediated injury, and hemodynamic instability leading to hypovolemia. $^{21}$

Liver enzymes were found elevated in both diseases with profound elevations noted in SGOT and SGPT levels in dengue. This corroborated with the results from prior studies ${ }^{25,26}$ which showed that, although hepatopathy in malaria was a common finding, elevations more than three times were seldom found. A significant $(\mathrm{P}<0.001)$ association between dengue and highly elevated ( $>3$ times) serum transaminases (SGOT and SGPT) has also previously been established by Jain et al. ${ }^{14}$ (74\% and $48 \%$ respectively) when comparing the disease with chikungunya. Moreover, hyperbilirubinemia in malaria, as also noticed in studies by Epelboin et al., ${ }^{8}$ Abhilash et al., ${ }^{12}$ and Chrispal et al. ${ }^{25}$ can be explained by the hemolysis occurring in malaria, ${ }^{21}$ which is absent in dengue.

The biggest strength of this study is a head-to-head comparison of two very common diseases in India. On a literature search, no prior study could be found comparing clinical details thoroughly, along with an extensive laboratory and a radiological workup, to distinguish dengue and malaria. This distinction could be of notable help in resource-limited setups, like rural India. However, this study has some limitations. Since all the patients were recruited from a single centre in North India, validation of these results from varied geographical locations may play a pivotal role in future studies. Moreover, a larger sample size could further enhance the credibility of this comparison.

\section{Conclusion}

This study highlights several clinical and laboratory parameters which could potentially distinguish between patients with dengue and those with malaria. These parameters could be vital in establishing diagnosis and initiating treatment earlier, hence improving patient outcomes significantly.

\section{Acknowledgements}

This research did not receive any specific grant from funding agencies in the public, commercial, or not-for-profit sectors.

\section{References}

1. WHO. Global Strategy for dengue prevention and control, 2012-2020. . [Internet]. Who.int., Available from. https://www.who.int/denguecontrol/9789241504034/ en/; 2019.

2. Global technical strategy and targets for malaria 2016-2030. . [Internet]. Who.int., Available from. http://apps.who.int/gb/ebwha/pdf_files/WHA68/A68_R2-en.pdf? ua $=1 ; 2019$.

3. Dengue and severe dengue. . [Internet]. Who.int., Available from. https://www.who $\mathrm{int} /$ news-room/fact-sheets/detail/dengue-and-severe-dengue; 2019.

4. Fact sheet about malaria. . [Internet]. Who.int., Available from. https://www.who. int/news-room/fact-sheets/detail/malaria; 2019.

5. [Internet] World Malaria Report 2018. World Health Organization; 2019. Available from https://www.who.int/malaria/media/world-malaria-report-2018/en/.

6. Bhatt S, Gething P, Brady O, et al. The global distribution and burden of dengue. Nature. 2013;496(7446):504-507.

7. National Vector Borne Disease Control Programme. URL for dengue, URL for malaria https://nvbdcp.gov.in/index4.php?lang $=1$ \&level $=0$ \&linkid $=431$ \&lid $=$ 3715https://nvbdcp.gov.in/WriteReadData/1892s/88572564451563538190.pdf.

8. Epelboin L, Boullé C, Ouar-Epelboin S, et al. Discriminating malaria from dengue fever in endemic areas: clinical and biological criteria, prognostic score and utility of the C-reactive protein: a retrospective matched-pair study in French guiana. PLoS Neglected Trop Dis. 2013;7(9):e2420.

9. Mørch K, Manoharan A, Chandy S, et al. Acute undifferentiated fever in India: a 
multicentre study of aetiology and diagnostic accuracy. BMC Infect Dis. 2017;17:665.

10. Anyanwu PE, Fulton J, Evans E, Paget T. Exploring the role of socioeconomic factors in the development and spread of anti-malarial drug resistance: a qualitative study. Malar J. 2017;16(1):203.

11. Potts JA, Rothman AL. Clinical and laboratory features that distinguish dengue from other febrile illnesses in endemic populations. Trop Med Int Health 2008;13(11):1328-1340.

12. Abhilash KPP, Jeevan JA, Mitra S, et al. Acute undifferentiated febrile illness in patients presenting to a tertiary care hospital in South India: clinical spectrum and outcome. J Global Infect Dis. 2016;8(4):147-154.

13. Barua A, Gill N. A comparative study of concurrent dengue and malaria infection with their monoinfection in a teaching hospital in Mumbai. $J$ Assoc Phys India. 2016;64(8):49-52.

14. Jain V, Jyotsana, Chopra A, et al. Clinicobiochemical difference of patients presenting with dengue and chikungunya during post-monsoon season. J Clin Diagn Res 2018;12(3):OC01-OC04.

15. Thomas EA, John M, Bhatia A. Cutaneous manifestations of dengue viral infection in Punjab (north India). Int J Dermatol. 2007;46(7):715-719.

16. Nawale A, Joshi M, Borde A. Role of ultrasound in dengue infection. Int J Sci Res. 2016:5(10):1478-1487.

17. Motla M, Manaktala S, Gupta V, et al. Sonographic evidence of ascites, pleurapericardial effusion and gallbladder wall edema for dengue fever. Prehospital Disaster Med. 2011;26(5):335-341

18. Murphy S, Cserti-Gazdewich C, Dhabangi A, et al. Ultrasound findings in plasmodium falciparum malaria: a pilot study. Pediatr Crit Care Med. 2011;12(2):e58-e63.

19. Santis OD, Kilowoko M, Kyungu E, Sangu W, Cherpillod P, Kaiser L. Predictive value of clinical and laboratory features for the main febrile diseases in children living in Tanzania: a prospective observational study. PloS One. 2017;12(5):e3314.

20. Kotepui M, PhunPhuech B, Phiwklam N, Uthaisar K. Differentiating between dengue fever and malaria using hematological parameters in endemic areas of Thailand. Infect Dis Poverty. 2017;6(27):1-9.

21. White NJ, Ashley EA. Malaria. In: Jameson JL, Kasper DL, Longo DL, Fauci AS, Hauser SL, Loscalzo J, eds. Harrison's Principles of Internal Medicine. $20^{\text {th }}$ ed. New York: McGraw-Hill; 2018:1575-1590.

22. Carme B, Sobesky M, Biard MH, Cotellon P, Aznar C, Fontanella JM. Non-specific alert system for dengue epidemic outbreaks in areas of endemic malaria. A hospitalbased evaluation in Cayenne (French Guiana). Epidemiol Infect. 2003;130(1):93-100.

23. Shah I, Katira B. Clinical and laboratory profile of dengue, leptospirosis and malaria in children: a study from Mumbai. Arch Dis Child. 2007;92(6):561

24. Nair JJ, Bhat A, Prabhu MV. A clinical study of acute kidney injury in tropical acute febrile illness. J Clin Diagn Res. 2016;10(8):OC01-OC5.

25. Chrispal A, Boorugu H, Gopinath KG, et al. Acute undifferentiated febrile illness in adult hospitalized patients: the disease spectrum and diagnostic predictors- an experience from a tertiary care hospital in South India. Trop Doct. 2010;40(4):230-234.

26. Kayley N, Ahmad S, Shirazi N, et al. Malarial hepatopathy: a 6-year retrospective observational study from Uttarakhand, North India. Trans R Soc Trop Med Hyg. 2017;111(5):220-225. 